\title{
Optimization of Machining Techniques in CNC Turning Centre Using Genetic Algorithm
}

\author{
H. Ganesan • G. Mohankumar
}

Received: 20 April 2011 / Accepted: 21 June 2011 / Published online: 1 February 2013

(C) The Author(s) 2013. This article is published with open access at Springerlink.com

\begin{abstract}
In a manufacturing industry, machining process is to shape the metal parts by removing unwanted material. During the machining process of any part given quality specifications such as surface finish, accuracy with minimum production cost or machining time are to be considered. Economy of machining operation plays a key role in competitiveness in the market. This paper presents a multi-objective optimization technique, based on genetic algorithms, to optimize the cutting parameters in turning processes: cutting depth, feed and speed. Optimization of cutting parameters is one of the most important elements in any process planning of metal parts. In this paper the three objective functions, minimum operating time and minimum production cost and minimum tool wear are simultaneously optimized. The proposed model uses a genetic algorithm in order to obtain the nondominated sorting genetic algorithm (NSGA-II) and build the Pareto front graph. An application sample is developed and its results are analyzed for several different production conditions. This paper also remarks the advantages of multiobjective optimization approach over the single-objective one.
\end{abstract}

Keywords Turning processes .

Multi-objective optimization .

Non-dominated sorting genetic algorithm

H. Ganesan $(\bowtie)$

Department of Mechanical Engineering,

RVS College of Engineering and Technology,

Coimbatore, Tamilnadu, India

e-mail: ganeshmelur@yahoo.co.in

G. Mohankumar

Park College of Engineering and Technology,

Coimbatore, Tamilnadu, India

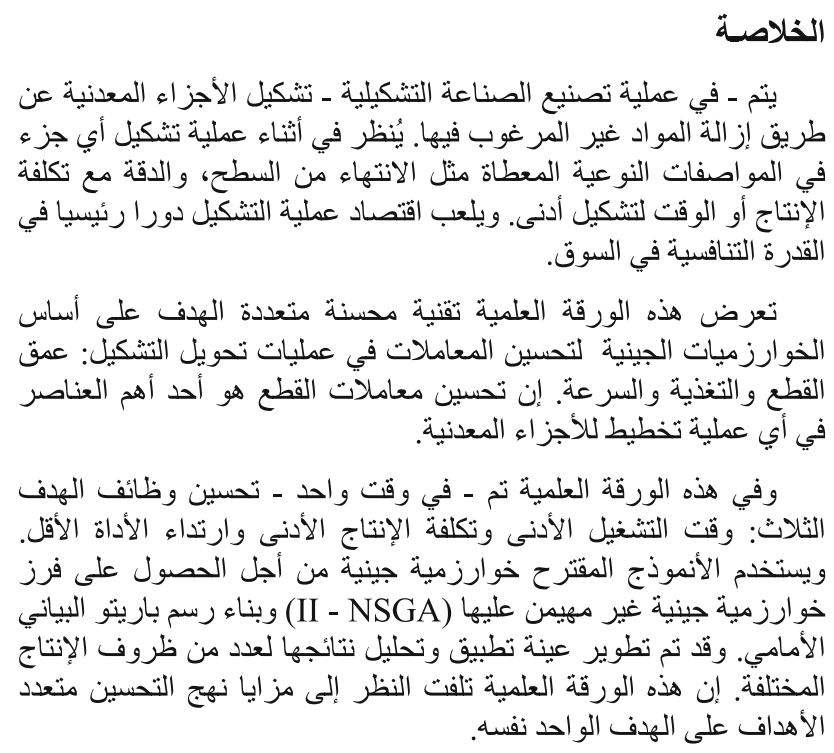

\section{Introduction}

Nowadays CNC machines are commonly used in industry. The operation of this machine is expensive because it has many parameters to consider. Optimization of cutting parameters is usually a difficult work where the following aspects are required: knowledge of machining; empirical equations relating the forces, power, surface finish, and dimensional accuracy etc. It has long been recognized that conditions during cutting, such as feed rate, cutting speed and depth of cut, should be selected to optimize the economics of machining operations, as assessed by productivity, and total manufacturing cost per component. The optimum machining conditions using Nelder-Mead simplex method was developed by Agapiou [1]. An objecting function incorporating 
a combination of minimum production cost and minimum production time and production cost is prioritized through their weight coefficients. A constant multiplier is used to normalize the objective function. Physical constraints regarding the cutting parameters are also considered. The superiority of the combined objective function over the single criterion objective functions using the production cost, or production time, or maximum profit rate is also illustrated. He also outlined the use of two techniques (Dynamic Programming and Nelder-Mead simplex method) to determine the optimum cutting conditions for the multipass operations. The optimum number of machining passes is obtained through the dynamic programming technique and the optimum machining conditions for each pass are then determined based on the combined objective function of total production cost and the total production time. This approach can be very effectively applied to the multistage machining since the optimum arrangement of the different operations can be determined by the dynamic programming method while the optimum cutting conditions for the operation in each machining stage are obtained using the method for single pass (Nelder-Mead simplex method) while incorporating the objective function.

Abburi and Dixit [2] proposed a methodology for the multi-objective optimization of multipass turning process. A real parameter genetic algorithm is used for minimizing the production time, which provides a nearly optimum solution. Kolahan and Abachizadeh [3] proposed the multipass turnings with seven different constraints are considered in a non-linear model where the goal is to achieve minimum total cost. Calculation of machining parameters in turning operation using machining theory was carried out by Meng et al. [4]. The objective criteria used in this work is minimum cost. Onwubolu et al. [5] have used a genetic algorithm for optimizing multipass turning operations. Saravanan et al. [6] considered the machining parameters optimization for turning cylindrical stock into a continuous finished profile using genetic algorithm (GA). Lee and Tarng [7] stated cutting-parameter selection for maximizing production rate or minimizing production cost in multistage turning operations. Sardinas et al. [8] expressed genetic algorithm-based multi-objective optimization of cutting parameters in turning processes to optimize the tool life and production rate. Gupta et al. [9] considered multipass turning optimization with optimal subdivision of depth of cut.

Bouzid [10] described for calculating the optimum cutting conditions in turning and the objective is to minimize the production time. The method uses empirical models for tool life, roughness and cutting forces. Arezoo et al. [11] stated a selection of cutting tools and conditions of machining operation to minimize production time. Ozel et al. [12] employed experimental observation and neural network modeling to evaluate the influences of cutting parameters on surface roughness of work piece machined. Chou and Song [13] determined the relation between tool nose radius and surface finish from experimental measurement. Chen [14] investigated the influence of cutting speed, tool wear and plastic behaviors of work piece material on surface finish. Decreasing tool nose radius and clearance angle can simultaneously decrease dimensional error and it is clear that the production of dimensional error in turning is possible. Choudhury and Srinivas [15] determined flank wear in turning operation. Huang and Liang [16] developed a model for tool flank wear in finish hard turning.

On the contrary, studies on evolutionary algorithms have shown that these methods can be efficiently used to eliminate most of the above-mentioned difficulties of the classical method. The proposed model uses a genetic algorithm in order to obtain the non-dominated sorting genetic algorithm (NSGA II) and build the Pareto front graph. NSGA II is widely used in defense and robotics field nowadays. For best movement of robot, the GA concept was used. By analyzing the best results in each movement by NSGA II concept, the robot feels the best value to move any direction. By keeping that value (non-dominated solutions) which was preserved by NSGA II, it will move at next time. In defense field, it will be used to attack the enemies with minimum time by giving the optimized speed and velocity value to the missile.

Most of the researchers used traditional optimization techniques for solving machining problems. The traditional methods of optimization are not efficient when the practical search space is too large. Traditional techniques such as geometric programming, dynamic programming find it hard to solve these problems, and they are inclined to obtain a local optimal solution. Considering the drawbacks of traditional optimization techniques, few researchers considered the non-traditional optimization techniques for the same problem domain.

Recently Karpet and Ozel [17] proposed multi-objective optimization for turning processes using neural network modeling and dynamic neighborhood particle swarm optimization and the objective function considered are minimum surface roughness and maximize productivity. The constraints considered are feed rate, cutting speed, depth of cut and tool geometry. Bharathi Raja and Baskar [18] discussed the optimization techniques for machining operations a retrospective research based on various mathematical models and testing with three different algorithms. The constrains considered are cutting speed, temperature, cutting force, power, feed and depth of cut. Yang and Natarajan [19] considered the multi-objective optimization of cutting parameters on turning process using differential evaluation and non-dominated sorting genetic algorithm approach to minimize the tool wear and maximize the metal removal rate and the constraints considered are temperature and surface finish.

Last year Datta and Majumder [20] suggested the optimization of turning process parameters using multi-objective 
evolutionary algorithm and the objective functions considered are tool life and operation time. The constraints considered are cutting force, surface roughness and power.

It is proposed to obtain the optimal parameters in turning processes to minimize the production cost, minimize the operation time and minimum tool wear simultaneously. In NSGA II the maintenance of an elitist population is a common technique to preserve the individuals. At the end of each evolutionary period, the non-dominated individuals are selected from the dynamic population and added to the elitist one. After the addition of new individuals, the elitist populations should be filtered in order to eliminate dominated individuals.

\section{Problem Formulation}

In $\mathrm{CNC}$ machine tools, the finished component is obtained by a number of rough passes and finish pass. To carry the rough and finishing operation it takes different speed, feed and depth of cut in our problem. By giving the optimized value of speed, feed and depth of cut, the production rate will increase with the minimum time, minimum cost and minimum tool wear. To obtain this objective we carried our problem by using NSGA II algorithm and produce the best results.

\subsection{Model for Machining Performance}

The first objective function is to minimize the production cost which includes machining cost, machine idle cost, the tool replacement cost and the tool cost. The formula for calculating the above cost is as given by $\mathrm{Chen}$ and $\mathrm{Su}$ [21]. Finally, by using the above mathematical processes, the unit production cost UC (Rs./piece) can be obtained as

$$
\begin{aligned}
\mathrm{UC} & =\mathrm{CM}+\mathrm{CI}+\mathrm{CR}+\mathrm{CT} \\
& \left.=k o T_{M}+k o T 1+k_{o} \| t_{o} T_{m} / t_{l}\right)+K_{t} T_{m} / t_{e}
\end{aligned}
$$

where

$$
\begin{aligned}
& \mathrm{CM}=\text { cutting cost (Rs./piece) } \\
& \mathrm{CI}=\text { machine idling cost (Rs./piece) } \\
& \mathrm{CR}=\text { tool replacement cost (Rs./piece) } \\
& \mathrm{CT}=\text { tool cost (Rs./piece) } \\
& k o=\text { sum of direct labour cost and overhead (Rs./min) } \\
& T_{M}=\text { actual cutting time } \\
& T I=\text { machine idle time (min) } \\
& t l=\text { tool life (min) } \\
& t e=\text { time required to exchange a tool (min) } \\
& k t=\text { cutting edge cost (Rs./edge) }
\end{aligned}
$$

The second objective function is to minimize the operation time, to measured as the entire time required to carry out the workpiece

$$
\begin{aligned}
t_{p} & =T_{m}+t_{e}\left(T_{m} / t_{l}\right)+\left[T_{i}\right] \\
& =\left(L_{j}+3 / f^{*} n\right)+t_{e}\left(\left(L_{j}+3 / f^{*} n\right) / f^{*} d^{*} n\right)+\left[T_{i}\right]
\end{aligned}
$$

where

$t_{p}=$ Unit time per work piece (min)

$T_{m}=$ Cutting/(machining time $)$ time

$t_{e}=$ Time required to exchange a tool (min)

$t_{l}=$ Tool life $(\mathrm{min})$

$T_{i}=$ Machine idle time

$L_{j}=$ Length of the travel

$f=$ feed

$n=$ speed

$d=$ depth of cut

The third objective function is to minimize the tool wear, The tool will be giving unsatisfactory performance during machining process due to wear and the tool is subjected to three important factors such as forces, temperature and sliding action which cause loss in dimensional accuracy, and increased surface roughness. This will result in loss of production and cost of replacing. To minimize the tool wear the empirical relation between tool wear and the machining variables is given by

$T_{w}=0.33349 V^{0.14804} f^{0.49116} d^{0.28979}$

\subsection{Machining Constraints}

The practical constraints imposed during the roughing and finishing operations are described below.

\subsubsection{Parameter Bounds}

Bounds on cutting speed:

$V_{r L} \leq V_{r} \leq V_{r U}$

where $V_{r L}$ and $V_{r U}$ are the lower and upper bounds of cutting speed in roughing, respectively.

Bounds on feed: $f_{r L}<f_{r}<f_{r U}$ where $f_{r L}$ and $f_{r U}$ are the lower and upper bounds of feed in roughing, respectively. Bounds on depth of cut: $d_{r L}<d_{r}<d_{r U}$ where $d_{r L}$ and $d_{r U}$ are the lower and upper bounds of depth of cut respectively.

\subsubsection{Cutting Force}

The expression for the cutting force constraint is given by

$F r=k f f r \int_{r}^{\mu} d \int_{r}^{v} \square \leq F_{U}$

where $F r$ is the cutting force during rough machining, $k f, \mu$ and $v$ are the constants pertaining to a specific tool-workpiece combination, and $F_{U}$ is the maximum allowable cutting force (kgf). 


\subsubsection{Power Constraint}

The power constraint is given by

$P_{r}=\frac{k f \int_{r}^{\mu} d \int_{r}^{v} V r}{6120 \eta} \leq P_{U}$

where $P_{r}$ is the cutting power during rough machining $(\mathrm{kW})$, $\eta$ is the power efficiency, and $P_{U}$ is the maximum allowable cutting power $(\mathrm{kW})$.

\subsubsection{Dimensional Accuracy Constraint}

The regressional relation for calculating the dimensional accuracy is given below:

$\delta=100.66 . f^{0.9709} d^{0.4905} V^{0.2848}$

where $\delta$ is the dimensional accuracy, $f$ is the feed rate per revolution, $d$ is the depth of cut, and $V$ is the cutting speed.

\subsubsection{Surface Finish Constraint}

The maximum allowable surface roughness is calculated as given below. Surface roughness is influenced by the feed and the nose radius of the tool:

$\frac{f s 2}{8 r} \leq R_{\max }$

where $r$ is the nose radius of cutting tool ( $\mathrm{mm}), R_{\max }$ is maximum allowable surface roughness $\left(\mu_{m}\right)$ and $f s$ is the feed.

\section{Solution Methodology}

Since traditional methods are not optimal to solve these problem. Hence, Genetic algorithms (GA) are a best population search based technique, GA are different from traditional optimizations in the following ways.

1. GA goes through solution space starting from a group of points and not from a single point.

2. GA search from a population of points and not a single point.

3. GA use information of a fitness function, not derivatives or other auxiliary knowledge.

4. GA use probabilistic transitions rules, not deterministic rules.

5. It is very likely that the expected GA solution will be a global solution.

The cutting conditions are encoded as genes by binary encoding to apply GA in optimization of machining parameters. A set of genes is combined together to form chromosomes, used to perform the basic mechanisms in GA, such as crossover and mutation. GA optimization methodology is based on machining performance predictions models developed from a comprehensive system of theoretical analysis, experimental database and numerical methods. The GA parameters along with relevant objective functions and set of machining performance constraints are imposed on GA optimization methodology to provide optimum cutting conditions.

\subsection{Genetic Algorithm Methodology}

Genetic algorithms are computerized search and optimization algorithms based on the mechanics of natural genetics and natural selection. Optimization can be done by the generation of the population.

\subsection{Steps in the Genetic Algorithm Optimization}

1. Choose a coding to represent problem parameters, a selection operator, a crossover operator, and a mutation operator. Choose population size $n$, crossover probability $p c$, and mutation probability $p m$. Initialize a random population of strings of size $l$. Choose a maximum allowable generation number $t_{\max }$. Set $t=0$.

2. Evaluate each string in the population.

3 . If $t t_{\max }$ or other termination criteria are satisfied, terminate.

4. Perform reproduction on the population.

5. Perform crossover on pair of strings with probability $p c$.

6. Perform mutation on strings with probability $\mathrm{pm}$.

7. Evaluate strings in the new population. Set $*=t+1$ and go to Step 3.

\subsection{Genetic Algorithm Parameters}

Population size: 32

Length of Chromosome: 6

Selection operator: Rank order

Crossover operator: Single point operator

Crossover probability: 0.75

Mutation probability: 0.1

Fitness parameter: Production cost, operation time and tool wear.

\subsection{Implementation of GA with Numerical Illustration}

Implementation plays an vital role in the genetic algorithm. A problem can be solved once it can be represented in the form of a solution string (chromosomes). The bits (genes) in the chromosome could be binary, real integer numbers. In this work, the cutting speed, feed rate and depth of cut are considered to be the primary parameters for the turning 
Table 1 GA implemented in unit cost, time and tool wear

\begin{tabular}{|c|c|c|c|c|c|c|}
\hline Speed (rpm) & Feed $(\mathrm{mm} / \mathrm{rev})$ & Depth of cut (mm) & Op time $(\min )$ & Unit cost (Rs) & Tool wear $(\mu)$ & Rank \\
\hline 1254.762 & 0.375 & 0.51 & 3.135 & 1.569 & 0.487 & 1 \\
\hline 1035.714 & 0.375 & 1.081 & 3.163 & 1.582 & 0.589 & 1 \\
\hline 980.952 & 0.177 & 0.357 & 3.371 & 1.7 & 0.293 & 1 \\
\hline 1254.762 & 0.128 & 1.119 & 3.397 & 1.704 & 0.36 & 1 \\
\hline 2842.857 & 0.029 & 1.119 & 3.788 & 1.916 & 0.195 & 1 \\
\hline 542.857 & 0.177 & 0.586 & 3.673 & 1.865 & 0.31 & 1 \\
\hline 2076.19 & 0.326 & 1.367 & 3.093 & 1.547 & 0.652 & 1 \\
\hline 1473.81 & 0.375 & 1.1 & 3.114 & 1.558 & 0.624 & 1 \\
\hline 2788.095 & 0.375 & 1.119 & 3.06 & 1.53 & 0.689 & 1 \\
\hline 2788.095 & 0.375 & 0.51 & 3.06 & 1.53 & 0.549 & 1 \\
\hline 1528.571 & 0.369 & 0.91 & 3.112 & 1.557 & 0.589 & 1 \\
\hline 1035.714 & 0.375 & 1.119 & 3.163 & 1.582 & 0.595 & 1 \\
\hline 3007.143 & 0.375 & 0.548 & 3.056 & 1.528 & 0.566 & 1 \\
\hline 1090.476 & 0.326 & 0.776 & 3.178 & 1.591 & 0.503 & 1 \\
\hline 3226.19 & 0.375 & 1.119 & 3.052 & 1.526 & 0.704 & 1 \\
\hline 2842.857 & 0.016 & 0.852 & 4.421 & 2.298 & 0.136 & 1 \\
\hline 1035.714 & 0.375 & 1.195 & 3.163 & 1.582 & 0.606 & 1 \\
\hline 1254.762 & 0.177 & 1.081 & 3.285 & 1.646 & 0.419 & 1 \\
\hline
\end{tabular}

operation. Each of these primary variables is represented in a binary string format. The total length of the string is 18 in which first 6 bits are used for speed representation and next 6 bits represent the feed variable and the remaining 6 bits are used as depth of cut parameter. The speed, feed and depth of cut are represented as substrings in the chromosome. The strings (000000 000000000000$)$ and (111111, 111111, 111111) represent the lower and upper limits of speed, feed and depth of cut.

\subsubsection{Initialization}

During initialization, a solution space of a "population size" solution string is generated randomly between the limits of the speed, feed and depth of cut. In this work the solution space size (population size) is considered as 18 as shown in Table 1. Columns 1, Column 2 and 3 show the initial random binary population. Column 4, 5, 6 show the objective function output (Optimized output). Column 7 denotes the Rank value. Here the binary format population can be decoded by using the below formula.

$x_{i}=x_{i}^{(L)}+\frac{x i(U)-x i(L)}{2 n-1} \quad($ decoded decimal value $)$

where $x i$ is the decoded speed or feed or depth of cut, $x(L) i$ is the lower limit of speed or feed or depth of cut, $x(U) i$ is the upper limit of speed or feed or depth of cut, and $n$ is the substring length (=6) (Fig. 1).

\subsubsection{Evolution}

In a GA, a fitness function value is computed for each string in the population, and the objective is to find a string with the maximum fitness function value. It is often necessary to map the underlying natural objective function to a fitness function form through one or more mappings. Since, we use a minimization objective function, the following transformation is used

$f(x)=\frac{1}{1+g(x)}$

where $g(x)$ is the objective function (operation time) and $f(x)$ is the fitness function. In the minimization problem the string which has the higher fitness value will be the best string.

\subsubsection{Selection and Reproduction}

Reproduction selects good strings in a population and forms a mating pool. The reproduction operator is also called a selection operator. In this work rank order selection is used. In Table 1 Column 4 shows the output generated using this method. Column 5 is the corresponding rank of the string. A lower ranked string will have a lower fitness value or a higher objective function and vice versa.

The higher cumulative probability value in the range is chosen as one of the parents. In Table 1 for the first string the generated random number is 0.237122 . The string number (rank) 1, which has a cumulative probability of 0.2485000 , 
Fig. 1 Block diagram of the proposed genetic algorithm

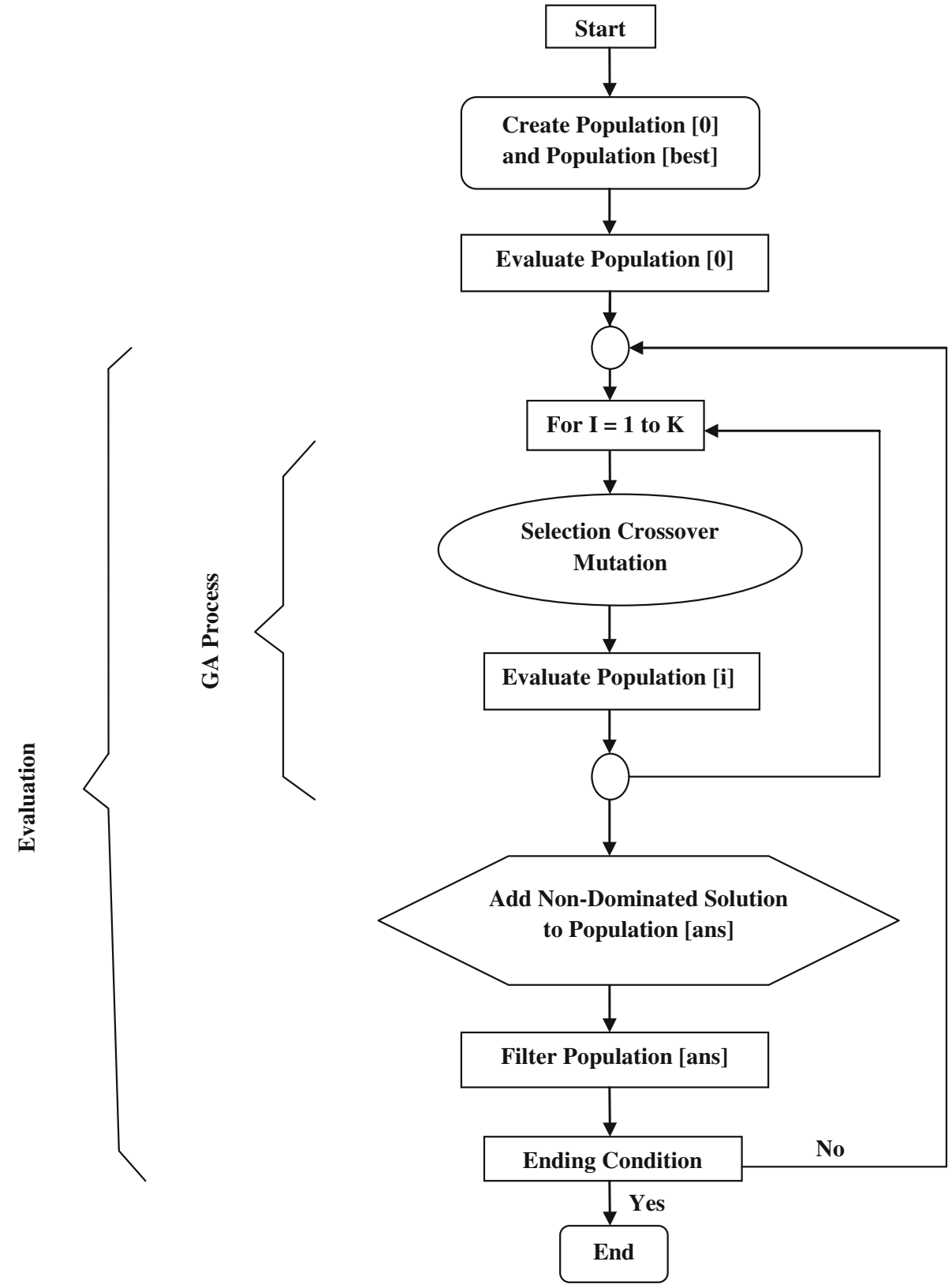

is selected as the parent, and this process is repeated for the entire population.

\subsubsection{Crossover}

Crossover is a mechanism for diversification. The strings to be crossed and the crossing points are selected randomly and crossover is done with a crossover probability. A single-point crossover is used in this work. The crossover probability is 0.75. The concept of crossover is explained below.

Before crossover:

1. $110010-00 \mid 0111$

2. $110100-01 \mid 0010$
After crossover: $1 \& 2 \rightarrow 9$ means crossover takes place between 1 st and 2 nd string at $(9+1)$ th cross site and after the $(9+1)$ th bit all the information is exchanged between strings. The cross site number starts from zero. Hence cross site number 9 represents the 10th site.

1. $110010-01 \mid 0111$

2. $110100-00 \mid 0010$

\subsubsection{Mutation}

Mutation is a random modification of a randomly selected string. Mutation is done with a mutation probability of 0.1 . 
Operation time Vs Unit Cost

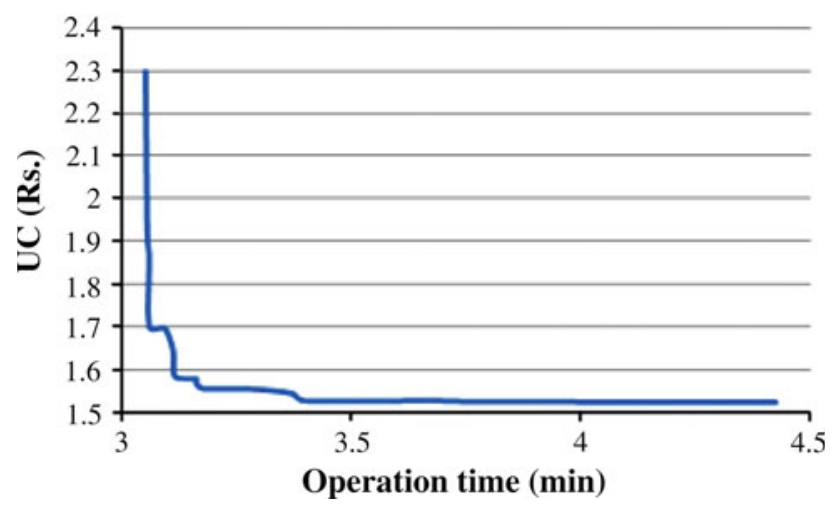

Fig. 2 Operation time (min) versus unit cost (Rs.)

Before mutation:

\section{11001001_0_111}

After mutation: $1 \rightarrow 9$ means that mutation takes place at the 1 st string at the 9 th site. The mutation will invert from 0 to 1 or 1 to 0 at the particular site.

\section{11001001_1_111}

The output after the first iteration is given in Table 1 . The best string in the list is the chromosome rank 1 which has minimum unit production cost. This completes one iteration of the GA and the best value is stored. All the strings available at the end of first iteration will be treated as parents for the second iteration. This procedure is repeated for the number of iterations as given by the user.

GA will give the best optimized results and time complexity of this algorithm is very less. It is a one of the algorithm, is mainly used as continuous process for the dynamic population.

\subsubsection{GA Implemented in Minimum Unit Cost and Production Time and Tool Wear}

\section{In this example,}

$x_{i}^{(L)}=50$ for speed $x_{i}^{(U)}=3500$ for speed

$x_{i}^{(L)}=0.01$ for feed $x i^{(U)}=0.4$ for feed

$x_{i}^{(L)}=0.3$ for depth of cut $x_{i}^{(U)}=1.5$ for depth of cut

Figure 2 indicates the Pareto front curve for operation time and production cost. It is evident that the cost is gradually decreasing up to 18th iteration and then the cost is constant for further iteration. Graph shows the minimum operation time for $4.421 \mathrm{~min}$ and the production cost is 1.526 . The curve also indicates the several different situations may be considered, facilitating the choice of right parameters for any condition.

Figure 3 indicates the Pareto front curve for unit cost and tool wear. It is evident that the cost is decreasing gradually and reaches the optimum value. The graph shows the
Toolwear Vs Unitcost

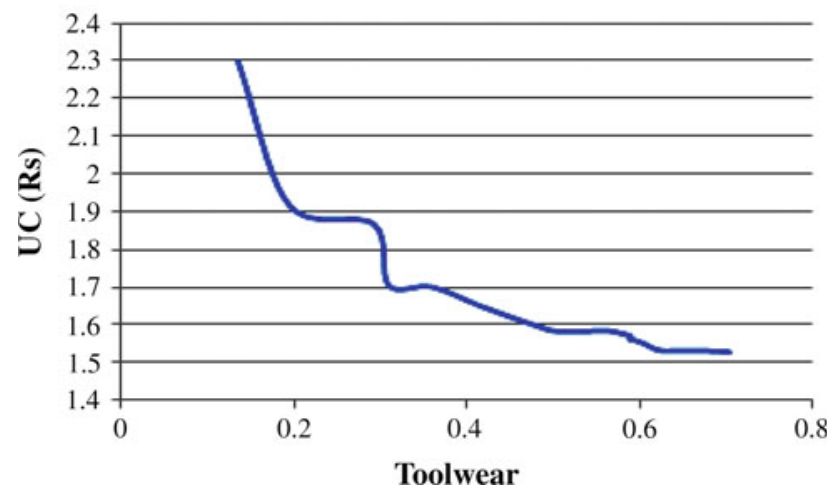

Fig. 3 Tool wear $(\mu)$ versus unit cost (Rs.)

Tool wear Vs Op Time

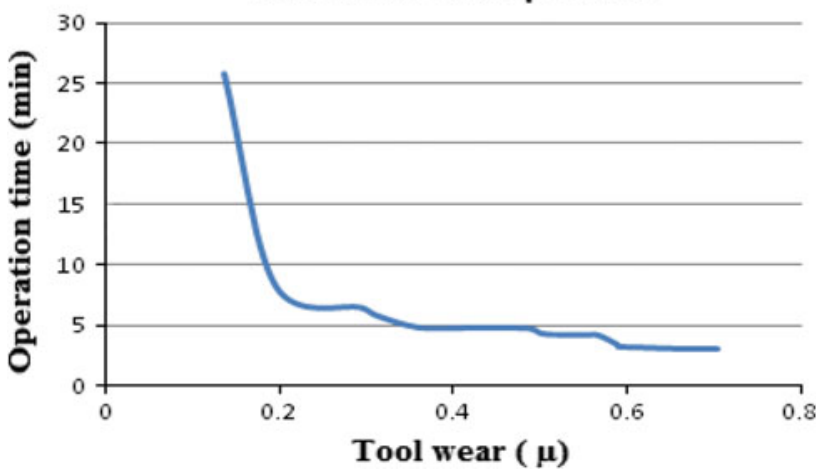

Fig. 4 Tool wear $(\mu)$ versus operation time (min)

optimum value of cost 1.569 and optimum tool wear 0.487 . It also indicates the several different combinations.

Figure 4 shows the Pareto front curve for operation time and tool wear. Fig shows clearly that the tool wear is decreased gradually in the initial iteration and then constant in further iteration. Graph shows the optimized minimum operation time $3.135 \mathrm{~min}$ and tool wear $0.487 \mu$. The curve also indicates and facilitating the choice of right parameter for any condition.

Figure 5 indicates clearly about the multi-objective optimization with minimum tool wear of 0.487 , operation time of 3.135 and production cost 1.569 . The graph shows the clear information about the optimization for different situation.

Figure 6 shows the optimum cutting parameters for optimum tool wear, operation time and cost. The curve facilitating the choice of right parameter for any condition.

\section{Test Example}

For testing the proposed methodology, the component shown in Fig. 7 is considered. The component is to be machined with optimal speed and feed using a SUPER JOBBER LM CNC 
Fig. 5 Optimization result of unit cost (Rs.), operation time $(\min )$ and tool wear $(\mu)$

Fig. 6 Optimization cutting condition

Fig. 7 Test component
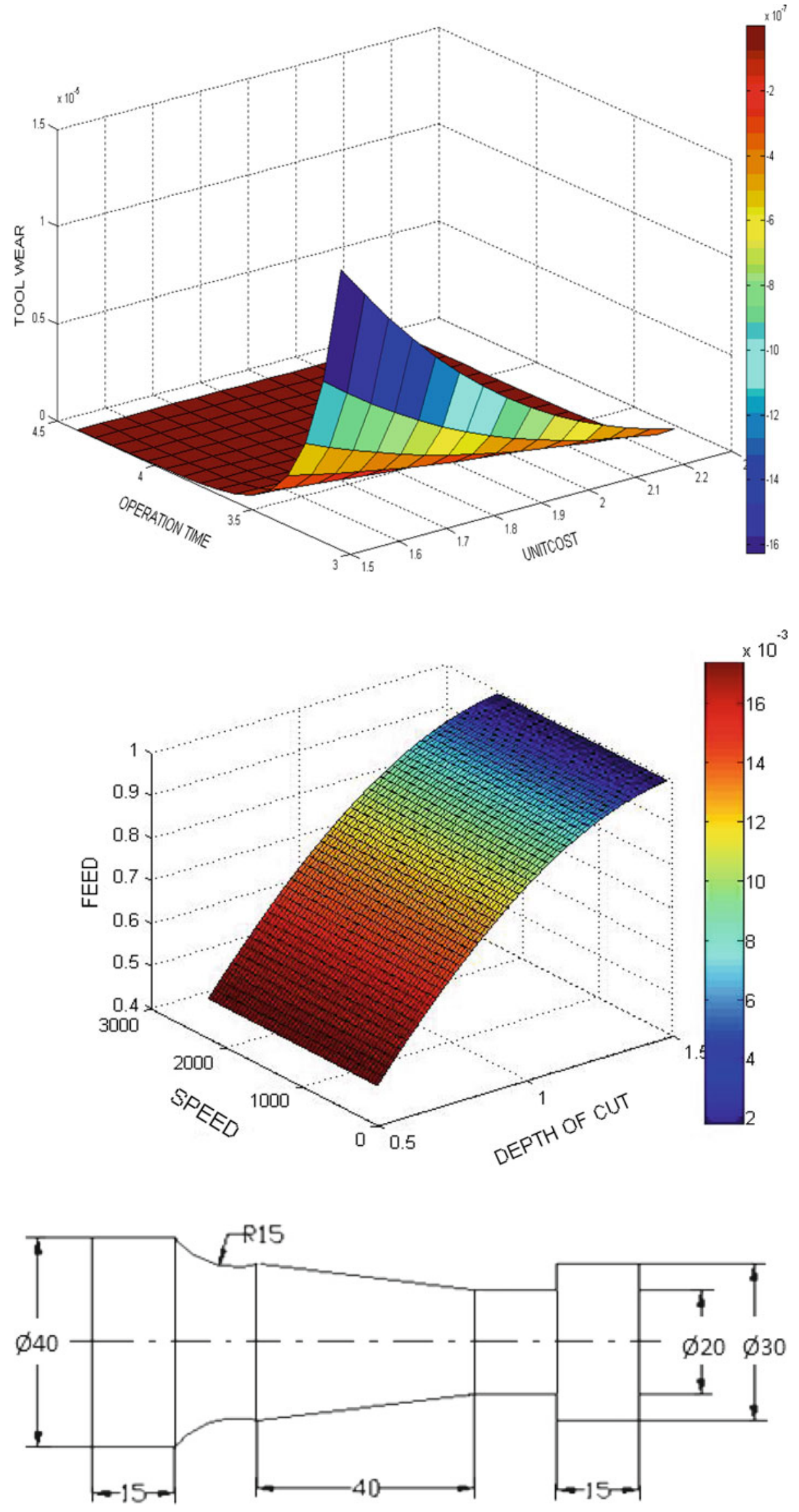
turning centre in the industry. The work piece material is EN 8 and the tool tip material is CNMG which is mostly used in rough turning and $0^{\circ}$ relief angle, provide longer life. The proposed model is run on an FANUC OI TD computer using the $\mathrm{C}++$ language. Tables and graphs summarize the computational results.

\section{Results and Discussion}

The results obtained from GA discussed here. By means of Pareto frontier graph several different situations may be considered in Fig. 2 shows the Pareto to obtain the optimal value and graph shows from point 1 to 7 decreases gradually and 8 to 18 only small variations. The Figs. 3 and 4 shows the optimal value between unit cost and tool wear, operation time and tool wear respectively and facilitating the choice of right parameters for any condition. Table 1 shows the optimal cutting parameters such as speed, feed and depth of cut obtained from GA for the minimum Operation time and minimum production cost and minimum tool wear. Figure 5 shows the fitness obtained in each iteration of the GA and shows the optimal time of $3.135 \mathrm{~min}$ and minimum cost of 1.569 and minimum tool wear of 0.487 . Figure 6 shows the optimum speed of $1254.762 \mathrm{rpm}$ and feed $0.375 \mathrm{~mm} / \mathrm{rev}$ and depth of cut $0.51 \mathrm{~mm}$. The above graphs shows that the GA produces smooth fitness at the initial iteration and varying fitness in the subsequent iterations. Using $\mathrm{C}++$ language the optimal solution can be obtained.

\section{Conclusion}

All types of $\mathrm{CNC}$ machines have been used to produce continuous finished profiles. A continuous finished profile has many types of operations such as facing, taper turning and circular turning. To model the machining process, several important operational constraints have been considered. These constraints were taken to account in order to make the model more realistic. The multi-objective optimization offers greatest amount of information in order to make a decision on selecting cutting parameters in turning. By means of Pareto graph several different situations may be considered, facilitating the choice of right parameters for any condition. The three dimension graph shows the additional information and the above said method can be improved further with additional constraints.

Open Access This article is distributed under the terms of the Creative Commons Attribution License which permits any use, distribution, and reproduction in any medium, provided the original author(s) and the source are credited.

\section{References}

1. Agapiou, J.S.: The optimisation of machining operations based on a combined criterion. Part 1: the use of combined objectives in single pass operations. ASME J. Eng. Ind. 114, 500-507 (1992)

2. Abburi, N.R.; Dixit, U.S.: Multi-objective optimization of multipass turning processes. Int. J. Adv. Manuf. Technol. 32, 902-910 (2007)

3. Kolahan, F.; Abachizadeh, M.: Optimizing turning parameters for cylindrical parts using simulated annealing method. In: Proceedings of World Academy of Science, Engineering and Technology, vol. 36, December 2008, ISSN: 2070-3740

4. Meng, Q.; et al.: Calculation of optimum cutting condition for turning operation using a machining theory. Int. J. Mach. Tool Manuf. 40, 1709-1733 (2000)

5. Onwubolu, G.C.; et al.: Multi-pass turning operations optimization based on genetic algorithms. Proc. Inst. Mech. Eng. 215, 117-124 (2001)

6. Saravanan, R.; et al.: Optimization of cutting conditions during continuous finished profile machining using non-traditional techniques to find the minimum production cost. Int. J. Adv. Manuf. Technol. 26, 30-40 (2005)

7. Lee, B.Y.; Tarng, Y.S.: Cutting-parameter selection for maximizing production rate or minimizing production cost in multistage turning operations. J. Mater. Process. Technol. 105(1-2), 61 - 66 (2000)

8. Sardinas, R.Q.: Genetic algorithm-based multi-objective optimization of cutting parameters in turning processes. Eng. Appl. Artif. Intell. 19, 127-133 (2006)

9. Gupta, R.; et al.: Determination of optimal subdivision of depth of cut in multi-pass turning with constraints. Int. J. Prod. Res. 33(9), 2555-2565 (1995)

10. Bouzid, W.: Cutting parameter optimization to minimize production time in high speed turning. J. Mater. Process. Technol. 161, 388-395 (2005)

11. Arezoo, B.; Ridgway, K.; AlAhmari, A.M.: Selection of cutting tools and conditions of machining operations using an expert system. Comput. Ind. 42, 43-58 (2000)

12. Ozel, T.; Karpat, Y.; Figueira, L.; Davim, J.P.: Modelling of surface finish and tool flank wear in turning of AISI D2steel with ceramic wiper inserts. J. Mater. Process. Technol. 189, 192-198 (2007)

13. Chou, Y.K.; Song, H.: Tool nose radius effects on finish hard turning. J. Mater. Process. Technol. 148, 259-268 (2004)

14. Chen, W.Y.: Cutting forces and surface finish when machining medium hardness steel using CBN tools. Int. J. Mach. Tools Manuf. 40, 455-466 (2000)

15. Choudhury, S.K.; Srinivas, P.: Tool wear prediction in turning. J. Mater. Process. Technol. 153, 276-280 (2004)

16. Huang, Y.; Liang, S.Y.: Modeling of CBN tool flank wear progression in finish hard turning. Trans. ASME 126(1), 98-106 (2004)

17. Karpat, Y.; Ozel, T.: Multi-objective optimization for turning processes using neural network modeling and dynamic-neighborhood particle swarm optimization. Int. J. Adv. Manuf. Technol. 35, 234247 (2007)

18. Bharathi Raja, S.; Baskar, N.: Optimization techniques for machining operations: a retrospective research based on various mathematical models. Int. J. Adv. Manuf. Technol. 48, 1075-1090 (2010)

19. Yang, S.H.; Natarajan, U.: Multi-objective optimization of cutting parameters in turning process using differential evolution and nondominated sorting genetic algorithm-II approaches. Int. J. Adv. Manuf. Technol. 49, 773-784 (2010) 
20. Datta, R.; Majumder, A.: Optimization of Turning Process Parameters Using Multi-Objective Evolutionary Algorithm. IEEE (2010)

21. Chen, M.C.; Su, C.T.: Optimization of machining conditions for turning cylindrical stocks into continuous finished profiles. Int. J. Prod. Res. 36(8), 2115-2130 (1998)
22. Deb, K.: Optimizations for Engineering Design-Algorithm and Examples. Prentice-Hall of India, New Delhi (1996) 